\title{
Unbalanced exchange flow and its implications for the night cooling of buildings by displacement ventilation
}

\author{
Nick Wise ${ }^{1}$ (D) Gary Hunt ${ }^{1}$
}

Received: 23 September 2020 / Accepted: 25 February 2021 / Published online: 19 March 2021

(c) The Author(s) 2021

\begin{abstract}
Passive ventilation of buildings at night forms an essential part of a low-energy cooling strategy, enabling excess heat that has accumulated during the day to self-purge and be replaced with cooler night air. Instrumental to the success of a purge are the locations and areas of ventilation openings, and openings positioned at low and at high levels are a common choice as there is then the expectation that a buoyancy-driven displacement flow will establish and persist. Desirable for their efficiency, displacement flows guide excess heat out through high-level openings and cooler air in through low-level openings. Herein we show that displacement flow cannot be maintained for the full duration of a purge. Instead, the flow must transition to an 'unbalanced exchange flow', whereby the cool inflow of air at low level is maintained but there is now a warm outflow and a cool inflow occurring simultaneously at the high-level opening. The internal redistribution of heat caused by this exchange alters the rate at which heat is self-purged and the time thought necessary to complete a purge. We develop a theoretical model that captures and predicts these behaviours. Our approach is distinct from all others which assume that a displacement flow will persist throughout the purge. Based on this enhanced understanding, and specifically that the transition to unbalanced exchange flow changes the rate of cooling and resultant emptying times, we anticipate that practitioners will be better placed to design passive systems that meet their target specifications for cooling.
\end{abstract}

Keywords Natural ventilation · Displacement flow $\cdot$ Balanced exchange flow $\cdot$ Unbalanced exchange flow $\cdot$ Mixing flow $\cdot$ Night cooling

\section{Introduction}

An important part of the summertime ventilation strategy of many naturally ventilated buildings is night cooling. This is the practice of utilising ventilation openings during the night, when the external air temperature is at its coolest, in order to purge the building of excess heat that has accumulated during the day. This purge also has the effect of cooling

Nick Wise

nhw24@cam.ac.uk

1 Department of Engineering, University of Cambridge, Trumpington Street, Cambridge CB2 1PZ, UK 


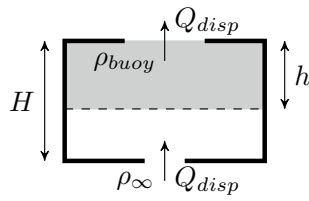

(a) Displacement flow

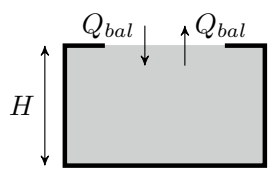

(b) Balanced exchange flow

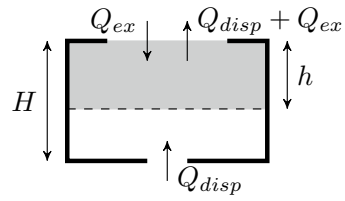

(c) Unbalanced exchange flow

Fig. 1 Schematics of three distinct buoyancy-driven flows in a room of height $H$ with high- and low-level openings, of areas $a_{p}$ and $a_{s}$, respectively, fully or partially filled with positively buoyant air of density $\rho_{\text {buoy }}$ (shaded grey) and air at density $\rho_{\infty}$. The room is surrounded by (night) air of density $\rho_{\infty}$. In a and c, $h$ denotes the instantaneous depth of the buoyant air layer. In $\mathbf{b}, a_{s} \equiv 0$

the thermal mass of the building, reducing radiative temperatures during the following day $[3,8]$.

The intended flow pattern for night cooling a single space, as opposed to one connected to others, e.g. by an atrium [17] or corridor, is typically either a displacement flow (Fig. 1a) or a balanced exchange flow-the latter also known as mixing flow (Fig. 1b). As will be shown and discussed herein however, a third flow pattern is possible, namely, that of unbalanced exchange flow (Fig. 1c). Whereas, with a displacement flow, there is equal flow in through the low-level opening and out through the high-level opening, and with a balanced exchange flow there is equal flow into and out of the high-level opening, with an unbalanced exchange flow the flow rates into and out of the high-level opening are not equal. There are well-established theoretical models for predicting the ventilation flow rates associated with displacement flow, for example [16], where the flow is driven by hydrostatic pressure differences, and balanced exchange flow [6], where the flow is driven by the Rayleigh-Taylor instability [21]. Unbalanced exchange flow, by contrast, has only been theoretically modelled recently [25] by considering the superposition of displacement flow and balanced exchange flow, in other words, by recognising that the overall flow comprises both displacement and exchange flow components.

In this paper we revisit the fundamental problem of predicting theoretically the night purging of a room by natural ventilation as, until now, a key aspect of the purge has been overlooked. We focus on what may be regarded as the simplest case, namely of a rectangular room with an opening positioned at high level to let heat out and an opening at low level to let cool night air in. At first sight, this configuration of openings would appear to drive a classic displacement flow and, thus, the problem would appear to have been solved previously. However, this is not the case, as during the purge a displacement flow (as depicted in Fig. 1a) must transition to an unbalanced exchange flow (as depicted in Fig. 1c). This transition was first identified by Hunt and Coffey [10] although the implications for a night purge had, until now, not been considered. As displacement flow cannot be maintained for the full duration of a purge, and specifically as following transition there is the unique situation of cool night air entering the room via both high-level and low-level openings, heat is redistributed internally. This redistribution then alters the rate at which heat is self-purged and, as a consequence, begs the question of how reliable displacement flow theory alone is for predicting the duration (and other characteristics) of a night purge. To address this question and, in the interests of developing a theoretical model that better describes an actual night purge, we develop herein a theoretical model that incorporates both the initial displacement flow phase and the subsequent unbalanced exchange flow phase.

After transition we apply the unbalanced exchange flow model of Wise and Hunt [25] and consider how it affects the predictions of existing simplified classic displacement 
flow. To do this we consider a 'typical' room of height $H(\mathrm{~m})$ and floor area $S\left(\mathrm{~m}^{2}\right)$, with one high-level horizontal opening and, in the case of displacement and unbalanced exchange flow, one low-level horizontal opening. The room is partially or fully filled with warm air to a depth of $h(\mathrm{~m})$. It is assumed that the characteristic lengthscale of the high-level opening is much greater than its wall thickness, such that the opening may be considered 'thin' (or sharp-edged), as in Wise and Hunt [25]. The low-level opening is treated in such a way that airflow velocities are small enough to prevent the incoming air, that rises upwards as a jet, from turbulently entraining warm air downwards across the density interface between the warm and cool air layers [10, 20].

While we draw from a number of key findings from the background literature, the overlap between the mathematical model for the complete purging of buoyant fluid from a box-like room developed in this paper and the previous works is relatively small and it is informative to make the distinctions clear. To the best of our knowledge, no one had previously developed a theoretical model for predicting how a room purges naturally that incorporates the flow transition from displacement to unbalanced exchange. Previous work had established:

(i) Under what conditions an exchange flow, balanced or unbalanced, must occur (Hunt and Coffey [10]) - their work on these conditions focusing on an examination of the flow exclusively at the opening,

(ii) What the flow rate associated with an unbalanced exchange must be based on the underlying instability (Wise and Hunt [25])—-their work focusing exclusively on the dynamics of the exchanging flow at the opening, and

(iii) Theoretical estimates for balanced exchange (Epstein [6])

Additionally, previous works had made measurements of:

(iv) Unbalanced exchange flow rates (Varral et al. [23]), and

(v) Balanced exchange flow rates (Epstein [6]).

Herein, we use the establish result (i) to inform at what stage our model for the purge should transition from displacement to unbalanced exchange flow, the results (ii)-(iii) to estimate the rate of fluid exchange during unbalanced exchange and the limit of balanced exchange, and (iv)-(v) together with established measurements of displacement flow to reason that we accurately capture the sequential stages that comprise a night purge.

The structure of the paper is as follows. First, in Sect. 2, the key non-dimensional parameters of the problem are introduced and discussed. The pertinent aspects of the established theories of displacement flow (Sect. 3) and balanced exchange flow (Sect. 4) are then revisited. In Sect. 5 we highlight a contradiction between these theories that underpins the aforementioned transition, then in Sect. 6 we discuss unbalanced exchange flow. In Sect. 7 we derive the governing equations for a room ventilated by displacement or unbalanced exchange flow and show that theoretically all night cooling displacement flows transition to unbalanced exchange, as well as considering the implications of this transition on the time taken to complete a purge. In Sect. 8 we investigate and highlight the stark benefits of adding even a small low-level opening to a room designed to utilise balanced exchange flow. Validation of the theory is considered in Sect. 9 and conclusions are drawn in Sect. 10. 


\section{Non-dimensional parameters}

For a general night cooling flow, be it displacement (Fig. 1a), balanced exchange (Fig. 1b) or unbalanced exchange (Fig. 1c), the airflow rates $\left(\mathrm{m}^{3} \mathrm{~s}^{-1}\right)$ are dependent on the buoyancy of the internal air relative to the external night air,

$$
g^{\prime}=g\left(\rho_{\infty}-\rho_{\text {buoy }}\right) / \rho_{\infty},
$$

In the definition of $g^{\prime}$, the quantity $\rho_{\text {buoy }}$, which may be a constant or a variable depending on the choice of openings, refers to the density of the warm air to be purged from the room, $\rho_{\infty}$ the density of the external night air, and $g$ the acceleration due to gravity. The density of the night air is assumed to be invariant throughout the duration of the night purge, i.e. we take $\rho_{\infty}=$ const. From the perspective of a practitioner wishing to work with air temperature $T$ (rather than air density), in the analysis that follows the density difference $\left(\rho_{\infty}-\rho_{\text {buoy }}\right)$ and reference density $\left(\rho_{\infty}\right)$ in (1) may be replaced by their respective temperatures, thus

$$
g^{\prime}=g\left(T_{\text {buoy }}-T_{\infty}\right) / T_{\infty}
$$

The natural choice of scale for non-dimensionalising the warm air layer depth, $h$ (Fig. 1 ), is the room height, $H$, and for the buoyancy is the value at the start of the purge, $g_{0}^{\prime}=g^{\prime}(t=0)$ where $t$ denotes time. Thus, we define

$$
\widehat{h}=h / H \quad \text { and } \quad \hat{g} \prime=g^{\prime} / g_{0}^{\prime}
$$

as the non-dimensional layer depth and buoyancy, respectively.

So as to be explicit regarding the area and position of ventilation openings, and the direction of flow through an opening, the following terminology and subscript convention is introduced with reference to a room of air that is warm, and therefore less dense, relative to the night air. The high-level opening is referred to as the primary opening, as only with a non-zero area is there flow under gravity. The low-level opening is referred to as the secondary opening. If the secondary opening area is zero, there is still a flow induced by the buoyancy force, although as this area is varied it conditions the flow through the primary opening. Quantities associated with the primary and secondary opening are labelled with the subscript $(\cdot)_{p}$ and $(\cdot)_{s}$, respectively. Similarly, airflow rates are designated by whether they are due to displacement, $(\cdot)_{\text {disp }}$, or to exchange, $(\cdot)_{e x}$, flow. In this context, we use 'disp' to refer to a unidirectional flow into the room through the secondary opening.

Two additional non-dimensional parameters are necessary to fully define the problem, for which we introduce the ratio of secondary to primary opening areas

$$
R=a_{s} / a_{p},
$$

and the ratio of the lengthscale of the primary opening to the room height

$$
\phi=\sqrt{a_{p}} / H .
$$

In identifying and classifying the different types of possible draining flow that may be anticipated during passive night cooling, including unbalanced exchange flow, Hunt and Coffey [10] introduced the parameter $\lambda=\sqrt{a_{p}} / h$ instead of $\phi$, however this would introduce the time dependent variable $h$ into the scaling, which is not of practical benefit here. The parameter $\lambda$ can be reconstructed simply from $\lambda=\phi / \hat{h}$. 
Dimensionless flow rates will henceforth be designated by

$$
\widehat{Q}=Q /\left(a_{p}^{5 / 4} g^{1 / 2}\right),
$$

in line with the notation used for dimensionless layer depth and buoyancy. For balanced exchange flow this is the only scaling possible on dimensional grounds, whilst for displacement flow and unbalanced exchange flow this scaling arises naturally as a ratio of the timescale for the growth of the instability that leads to exchange and the advection timescale. Further discussion may be found in Wise and Hunt [25]. Dimensionless time is defined as

$$
\widehat{t}=t / t_{\text {disp }},
$$

where $t_{\text {disp }}$, defined in Sect. 3, is the time predicted for a room to purge fully of warm air (i.e. the emptying time) based on the assumption that a displacement flow is established and maintained.

While flow rates have been normalised with respect to scalings pertinent to exchange flow, time has intentionally been normalised based on the scaling pertinent to displacement flow. The reasons are two fold. First, this scaling enables direct comparisons to be made with the emptying time according to classic displacement flow theory. Second, we are primarily interested in room ventilation that commences by displacement flow, a flow that we argue (Sect. 7) must transition to unbalanced exchange flow. We anticipate that for typical rooms, a displacement flow will be maintained for the majority of the purge duration.

We now consider each of the flows depicted in Fig. 1 in turn, the review of displacement (Sect. 3) and balanced exchange (Sect. 4) flows placing the new work in context. Beyond a scene setting, Sect. 4 extends our understanding of the balanced exchange flow case by considering the extent of mixing between the incoming night air and the air within the room. The simplified theoretical bounds we thereby place on the perfect mixing and no mixing cases are then used in Sects. 7 and 8 to investigate the limits of the effect of unbalanced exchange flow on night cooling.

\section{Displacement flow}

With an idealised displacement flow (Fig. 1a), positively buoyant air of density $\rho_{\text {buoy }}$ is exhausted naturally via the primary opening and replaced with air at ambient density $\rho_{\infty}$ via the secondary opening. Flow is assumed to be unidirectional through primary and secondary openings, and there is assumed to be negligible mixing ${ }^{1}$ at the horizontal interface between the warm air region and the inflow of air at ambient density through the secondary opening.

Assuming an incompressible flow, conservation of volume applied to a control volume coincident with the perimeter of the room requires

$$
Q_{\text {disp }}=Q_{s}=Q_{p}
$$

For small density differences (i.e. $\rho_{\infty}-\rho_{\text {buoy }} \ll \rho_{\infty}$ ), as are anticipated for a typical night purge, and on the assumption that there are negligible heat transfers with the fabric of the

\footnotetext{
${ }^{1}$ Hunt and Coffey [10] identify the constraints on the warm air layer depths and areas of primary and secondary opening for which the assumptions of unidirectional flow and negligible mixing are valid.
} 
room, the instantaneous volume flow rate driven by a two-layer displacement flow (Fig. 1a) is given by

$$
Q_{\text {disp }}=\underbrace{\left(\frac{1}{2 c_{s}^{2} a_{s}^{2}}+\frac{1}{2 c_{p}^{2} a_{p}^{2}}\right)^{-1 / 2}}_{\text {Effective opening area } A^{*}}\left(g^{\prime} h\right)^{1 / 2},
$$

where $c_{s}$ and $c_{p}$ are loss coefficients associated with flow through the secondary and primary openings, respectively (see, for example, $[13,16]$ ). In order to simplify the analysis we take $c_{s}=c_{p}=1$, equivalent to taking $a_{s}$ and $a_{p}$ as the effective areas of the openings [14]. For first-order predictive purposes, in line with much of the building ventilation literature it would not appear unreasonable to take $c_{p}=c_{s}=0.6$, however whilst these coefficients are well defined for unidirectional flow through an opening [8], it is not clear what is meant by a loss coefficient for an opening with bi-directional flow, such as balanced or unbalanced exchange flow.

It is thus worthy of note that for an idealised displacement flow, the room openings are characterised solely by the 'effective opening area' $A^{*}$ (the underbraced term in (9)) and consequently that, as recognised by [10], this theory would suggest that interchanging the areas of primary and secondary openings (so that the area ratio changes from $R$ to $1 / R$ ) results in an identical effective opening area-in other words, the area ratio is unimportant and does not play a role in characterising the purge. Hunt and Coffey [10] showed in their flow visualisations that this is not the case as changing $R$ to $1 / R$ whilst keeping $A^{*}$ fixed can have a profound effect on the flow and we show that this is not the case for a true purge as the flow must transition from displacement flow.

Non-dimensionalising (9), we find

$$
\widehat{Q}_{\text {disp }}=\frac{Q_{\text {disp }}}{a_{p}^{5 / 4} g^{1 / 2}}=\left(\frac{2 \hat{h}}{\phi}\right)^{1 / 2}\left(1+\frac{1}{R^{2}}\right)^{-1 / 2} .
$$

As shown by Wise and Hunt [25] and discussed in Sect. 6, non-dimensionalising the displacement flow rate by $a_{p}^{5 / 4} g^{1 / 2}$ gives the governing parameter for unbalanced exchange flow [6], and this is also the scaling that arises naturally for balanced exchange flow. This provides further justification for regarding the high-level opening of the room as the primary opening, and the low-level opening as secondary.

An implication of (9) is that for $a_{s} \ll a_{p}$, equivalent to $R \ll 1$, the displacement flow rate becomes

$$
Q_{\text {disp }}=2^{1 / 2} a_{s}\left(g^{\prime} h\right)^{1 / 2}+O\left(a_{s}^{3}\right)
$$

indicating that the flow rate is controlled primarily by the secondary opening area, the larger area of the primary opening having a second order effect. Therefore, (9) would suggest that with the primary opening open $\left(a_{p}>0\right)$, the ventilation of the room reduces to zero, i.e. $Q_{p}=Q_{s}=Q_{\text {disp }} \rightarrow 0$ as $a_{s} \rightarrow 0$. Of course, this can not be the case in practice.

If we consider the night cooling scenario of a room with floor plan area $S$ independent of height, initially full of air of density $\rho_{\text {buoy }}$ and containing no active heat sources or cooling sources other than the night air, the fractional depth of the buoyant layer as a function of time can be shown [16] to be 


$$
\widehat{h}=\left(1-\frac{t}{t_{\text {disp }}}\right)^{2},
$$

where

$$
t_{\text {disp }}=2^{1 / 2} \frac{S}{a_{p}}\left(1+\frac{1}{R^{2}}\right)^{1 / 2}\left(\frac{H}{g_{0}^{\prime}}\right)^{1 / 2}
$$

is the timescale for night cooling with displacement flow. Defining $t_{E}$ as the time taken for the room to fully empty of buoyant air (i.e. the emptying time), then beyond a timescale, (13) is the emptying time if an idealised displacement flow is maintained throughout the purge, i.e. for displacement flow $t_{E}=t_{\text {disp. }}$. Given, for an idealised displacement flow there is no mixing across the thermal interface, the density and therefore the buoyancy of the layer is unchanged over the entire duration of the purge, i.e. $g^{\prime}=g_{0}^{\prime}$ from $t=0$ to $t=t_{E}$. Substitution of $\hat{h}$ from (12) into (10) gives

$$
\widehat{Q}_{\text {disp }}=\left(\frac{2}{\phi}\right)^{1 / 2}\left(1+\frac{1}{R^{2}}\right)^{-1 / 2}\left(1-\frac{t}{t_{\text {disp }}}\right),
$$

showing that the displacement flow rate decreases linearly with time, until at $t=t_{\text {disp }}=t_{E}$ the flow rate $\widehat{Q}_{d i s p}=0$ and the purge is complete.

\section{Balanced exchange flow}

Balanced exchange concerns the flow that is established when the secondary opening is closed, so that $a_{s}=0$ and thus $R=0$ (Fig. 1b). This opening configuration means there is only a flow of air through the primary opening, where both inflow and outflow must occur simultaneously. This would occur, in practice, if low-level openings were closed during the day, for example, due to external air pollution, or at night for security reasons. The inflow and outflow are equal in magnitude to conserve volume and, hence, the exchange is referred to as balanced. Air at ambient density that now enters the room is negatively buoyant relative to the local environment and is observed in analogue flow visualisation experiments to drive vigorous mixing $[6,10,23]$. In contrast to an idealised displacement flow, the requirement for volume conservation means that, in the absence of interfacial mixing, the buoyant layer depth $h$ will not change with time, whereas the buoyancy of the air in the layer will reduce.

Whilst we only consider rooms which are initially full of buoyant air, so that $h=H$ at $t=0$, if we were to consider a partially filled room, i.e. so that $0<h<H$ at $t=0$, then the depth of the buoyant layer may increase with time. The incoming ambient air will fall until it impinges on the interface; if that air is sufficiently energetic it can cause a net transfer of air from below the interface into the buoyant layer above by a process of turbulent entrainment, thereby increasing the layer depth. Shrinivas and Hunt [20] develop a theoretical model for predicting the rate of turbulent entrainment in terms of the details of the impinging flow at the interface within the confining geometry of the room, however, in the interests of developing a simplified model, we shall not include such a vertical transport of fluid. 
The behaviour of balanced exchange flow in a saline-filled box representing a room initially filled with buoyant air, with a single opening in the top, was investigated by Epstein [6] for circular openings and Brown, Wilson and Solvason [1] for square openings. For socalled 'sharp-edged' openings, such as those we restrict our attention to herein, the volume flow rate either into, or out of, the opening was determined to scale as

$$
Q_{b a l}=k a_{p}^{5 / 4} g^{1 / 2} \quad \Longrightarrow \quad \widehat{Q}_{b a l}=k
$$

The value of the dimensionless factor $k$ has been determined by experimental measurement to be $k=0.05$ for circular openings [6] and $k=0.07$ for square openings [1]. The physical mechanism for initiating and maintaining the exchange was attributed to the instability of the interface between the buoyant air below the primary opening and the denser external night air above [6].

\subsection{Perfect mixing}

If we return to the night cooling scenario of a room of buoyant air with density $\rho_{\text {buoy }}(0)$ at the start of the purge but now with $a_{s} \equiv 0$ so that there is balanced exchange flow, the buoyancy of the air in the room as a function of time can be shown [16] to be

$$
\hat{g}^{\prime}=\frac{g^{\prime}}{g_{0}^{\prime}}=\left(1+\frac{t}{t_{b a l}}\right)^{-2},
$$

where the timescale for night cooling with balanced exchange

$$
t_{\text {bal }}=\frac{2 S H}{k a_{p}^{5 / 4} g_{0}{ }^{1 / 2}} \quad \text { with } \quad g_{0}^{\prime}=\frac{g}{\rho_{\infty}}\left(\rho_{\infty}-\rho_{\text {buoy }}(0)\right) .
$$

This solution assumes 'perfect' mixing, i.e. that the incoming air instantaneously mixes throughout the room so that the room air is always of uniform buoyancy. With this assumption, $h=1 \forall t>0$, i.e. unlike the displacement flow case (12), the room will never empty of buoyant air. Instead, the buoyancy of the air will simply decrease for all time. Given the volume $V$ of the room is $V=S H$, note from (17) that the timescale for the reduction in buoyancy, $t_{b a l}$, is linearly dependent on the room volume.

\subsection{No mixing}

If no mixing is assumed for a balanced exchange flow (whether neglecting mixing due to the inflow descending through the buoyant air layer or to its impingement with the interface) then the incoming cool night air will fall under the action of gravity to the floor of the room, form a layer and displace the buoyant air upwards and out of the room. However, whereas the volume flow rate of a displacement flow depends on both the reduced gravity and the depth of the buoyant layer, here the volume flow rate depends only on the reduced gravity local to the primary opening. In the absence of mixing, the reduced gravity is constant and therefore so is the volume flow rate. Conservation of volume then gives 


$$
\widehat{h}=1-2 \frac{t}{t_{b a l}}
$$

The emptying time, corresponding to $\widehat{h}=0$, is thus $t_{E} / t_{b a l}=1 / 2$. However, as we will now demonstrate in Sect. 4.3, balanced exchange flows in an unstratified room are expected to exhibit behaviour closer to perfect mixing than to no mixing.

\subsection{Imperfect mixing}

Experimental work [4, 16] has shown that the purge of a room, initially full of warm air, with balanced exchange flow is faster than that predicted by (16). Coffey and Hunt [4] attributed this difference to the internal buoyancy distribution not being uniform in practice, i.e. there being a vertical variation of buoyancy despite the vigorous mixing produced by the inflow. In practice, as the incoming cool night air (with buoyancy $g^{\prime}=0$ on entering the room, as defined) falls, it entrains buoyant air and its buoyancy increases towards that of the room air; once this descending cool plume-like flow of air reaches the floor level it spreads laterally to form a layer of intermediate density, cf. Hunt and Kaye [15]. The resulting non-uniformity in the room buoyancy means that, in practice, the air being exhausted is expected to be more buoyant than the mean buoyancy of the room and, therefore, buoyancy is purged faster than is assumed in (16).

If we assume that the incoming air descends to form a classic turbulent plume [18], i.e. with buoyancy varying as a function of distance $\widehat{z}=z / H$ from its source at the plane of the primary opening according to the power law $G^{\prime}=g\left(\rho_{\text {plume }}-\rho_{\text {buoy }}\right) / \rho_{\infty} \propto \widehat{z}^{-5 / 3}$, then the buoyancy of the incoming air when it has reached floor level may be approximated by

$$
\frac{\left.G^{\prime}\right|_{\hat{z}=1}}{\left.G^{\prime}\right|_{\hat{z}=0}}=\frac{\beta \phi^{5 / 3}}{\left(1+\beta^{3 / 5} \phi\right)^{5 / 3}},
$$

where $\beta$ is a constant taken to be $\beta=3$ and $\rho_{\text {plume }}$ denotes the local density of the plume. The derivation of (19) and reasoning behind taking $\beta=3$ are given in the Appendix. The limits of (19) are $\left.G^{\prime}\right|_{\hat{z}=1} /\left.G^{\prime}\right|_{\hat{z}=0}=0$, perfect mixing, and $\left.G^{\prime}\right|_{\hat{z}=1} /\left.G^{\prime}\right|_{\hat{z}=0}=1$, no mixing. By estimating $\left.G^{\prime}\right|_{\hat{z}=1} /\left.G^{\prime}\right|_{\hat{z}=0}$ we can assess whether perfect or no mixing is the better model for a given situation. If the calculated value of $\left.G^{\prime}\right|_{\hat{z}=1} /\left.G^{\prime}\right|_{\hat{z}=0}$ is closer to 0 , then perfect mixing is the better approximation; if the value is closer to 1 then no mixing is better.

In order to have perfect mixing between the incoming air and the air in the room, (19) shows that we would need $\phi=0$. Given $\phi=\sqrt{a_{p}} / H, \phi=0$ can only be achieved in the limit of an infinitely tall room or for the equally impractical limiting case of zero ventilation $\left(a_{p} \rightarrow 0\right)$. As $\phi$ increases so does the ratio $\left.G^{\prime}\right|_{\hat{z}=1} /\left.G^{\prime}\right|_{\hat{z}=0}$, with $\left.G^{\prime}\right|_{\hat{z}=1} /\left.G^{\prime}\right|_{\hat{z}=0} \rightarrow 1$ in the limit as $\phi \rightarrow \infty$. For a typical room with $H=3 \mathrm{~m}$ and square openings with $a_{p}=1 \mathrm{~m}^{2}$ then $\phi=1 / 3$. These values give $\left.G^{\prime}\right|_{\hat{z}=1} /\left.G^{\prime}\right|_{\hat{z}=0}=0.2$ to 1 significant figure (s.f.), therefore the assumption of perfect mixing is not unreasonable for a typical room and it provides a conservative upper bound for the calculation of emptying times.

For a two-layer stratified room however, the descending plume of night air will only fall to the level of the interface that separates the layers at $\widehat{z}=\widehat{h}$, before spreading laterally outward to form a new layer of intermediate density. Equation (19) is readily adapted to model this stratified case, giving 


$$
\frac{\left.G^{\prime}\right|_{\widehat{z}=\hat{h}}}{\left.G^{\prime}\right|_{\hat{z}=0}}=\frac{\beta \phi^{5 / 3}}{\left(\widehat{h}+\beta^{3 / 5} \phi\right)^{5 / 3}} .
$$

If the aforementioned room with $\phi=1 / 3$ were not full of buoyant air but instead had a shallow buoyant layer of depth $\hat{h}=0.1$, then $\left.G^{\prime}\right|_{\hat{z}=0.1} /\left.G^{\prime}\right|_{\hat{z}=0}=0.8$ and it would be more reasonable to assume no mixing at all.

It should be noted that the analysis that underlies (19) and (20) excludes any effect due to the outflow moving past the inflowing plume flow that has been assumed and any additional mixing that might generate. The analysis of the 'real' scenario with the formation of a layer of intermediate density and a plume that develops from non-steady source conditions is beyond the scope of this paper, our focus being on the development of a simplified model for the night purge that incorporates the observed transition in the behaviour of the flow at the primary opening.

\section{A contradiction indicating a third type of flow}

The theories for idealised displacement and balanced exchange flows are long established $[6,16]$, however there is a clear contradiction between the theory of displacement flow, which predicts that the ventilation flow rate $\widehat{Q}_{p}=\widehat{Q}_{s}=\widehat{Q}_{\text {disp }} \rightarrow 0$ as $a_{s} \rightarrow 0$, and that of balanced exchange flow which, in the same limit, predicts a non-zero volume flow rate of $\widehat{Q}_{p}=\widehat{Q}_{b a l}=k>0$ through the primary opening. This contradiction suggests that flows are not described well by displacement flow theory as $a_{s} \rightarrow 0$ and that there is an intermediate range of behaviour between the two limits of displacement flow and balanced exchange flow. We will now show that this contradiction is resolved by unbalanced exchange flow (Fig. 1c).

\section{Unbalanced exchange flow}

Unbalanced exchange flow shares features of both displacement and balanced exchange flow; there is inflow through the secondary opening (cf. displacement flow) as well as simultaneous inflow and outflow through the primary opening (cf. balanced exchange flow). For the night cooling scenario, this means that both the layer depth and buoyancy will vary with time during a purge. A theoretical explanation of unbalanced exchange flow and prediction of the flow rates and transition from displacement flow have recently been provided by Wise and Hunt [25]. Their work informs the modelling of a night purge developed herein and the discussion that follows.

\subsection{Transition from displacement to unbalanced exchange}

Consider a room, initially full of buoyant air, with a high-level primary opening and lowlevel secondary opening of equal area, i.e. of a geometry that one would expect to give rise to a displacement flow. If the area of the secondary opening is reduced, $R$ decreases, and hence, the flow rate will reduce (10). Consequently, the flow velocity through the primary opening will also reduce. If we continue reducing the area of the secondary opening, there will come a point where the flow velocity through the primary opening is so low 
that it is comparable with the velocities of the instability driving the exchange flow, allowing the instability to grow against the displacement flow and for ambient air to enter the room through the primary opening [25]. Denoting the critical flow rate at which this flow transition occurs as $Q_{C}$, we note there is displacement flow for $Q_{s}>Q_{C}$ and unbalanced exchange flow for $Q_{s}<Q_{C}$ (providing, of course, $a_{s} \neq 0$ ); with reference to (9) we note that $Q_{s}=Q_{\text {disp }}$ up to the very point of transition. While we have reasoned above that this transition can be triggered on reducing $a_{s}$ and, hence, the flow velocity through the primary opening, transition can also occur as this velocity reduces due to a reduction in the head of fluid driving the motion [10]; it is the latter that is instrumental to the transition from displacement to unbalanced exchange flow that we model in Sect. 7.

It should be noted that the theory of unbalanced exchange flow described by Wise and Hunt [25] includes no hysteresis. An identical critical flow rate is predicted whether the flow rate of a unidirectional flow through an opening is reduced until exchange initiates, or the flow rate of a unidirectional flow imposed on a balanced exchange flow is increased from zero until there is no exchange. This matches the experimental work [7, 23], which did not observe hysteresis.

The critical dimensionless displacement flow rate, $\widehat{Q}_{C}=Q_{C} / a_{p}^{5 / 4} g^{1 / 2}$, below which unbalanced exchange initiates was found to be

$$
\widehat{Q}_{C}=0.33 \text { experimentally [10] and } \widehat{Q}_{C}=0.29 \text { theoretically [25]. }
$$

For square openings, the theoretical prediction is $\widehat{Q}_{C}=0.22$ [25], though we are not aware of experimental measurements against which to compare this value.

\subsection{Flow rates for unbalanced exchange}

In the context of unbalanced exchange, and with reference to Fig. 1c, the distinction between the flow rates $Q_{d i s p}$ and $Q_{e x}$ may be understood as follows: $Q_{d i s p}$ is the inflow volume flow rate through the secondary opening driven by the stack pressures within the room and thus $Q_{s}=Q_{\text {disp }}$, whereas $Q_{e x}$ is inflow volume flow rate through the primary opening due to unbalanced exchange; as a consequence, the net volume flow rate out through the primary opening is the quantity $Q_{\text {disp }}$.

In between the limits that correspond to the onset of exchange (when $\widehat{Q}_{s}=\widehat{Q}_{C}$ ) and to balanced exchange flow (when $\widehat{Q}_{s}=0$ ), there is a single value of the volume flow rate exchanged $\widehat{Q}_{e x}$ for each value of $\widehat{Q}_{s}=\widehat{Q}_{\text {disp }}$, a value that depends on the opening geometry. This relationship between $\widehat{Q}_{\text {disp }}$ and $\widehat{Q}_{e x}$ is plotted in Fig. 2 for both circular and square openings. The functions plotted are analytic but are calculated from implicit equations so can not be stated simply; details of their derivation can be found in [25]. The relationships plotted, namely,

$$
\widehat{Q}_{e x}\left(\widehat{Q}_{\text {disp }}\right)=-0.81 \widehat{Q}_{\text {disp }}^{3}+1.14 \widehat{Q}_{\text {disp }}^{2}-0.43 \widehat{Q}_{\text {disp }}+0.055 \quad \text { (circular openings) }
$$

and

$$
\widehat{Q}_{e x}\left(\widehat{Q}_{\text {disp }}\right)=-2.4 \widehat{Q}_{\text {disp }}^{3}+2.5 \widehat{Q}_{\text {disp }}^{2}-0.78 \widehat{Q}_{\text {disp }}+0.076 \quad \text { (square openings) }
$$

agree well with all the available experimental data of [7] and [23]. 


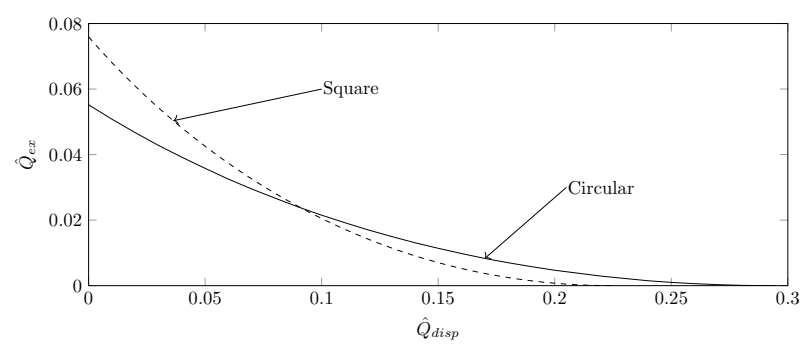

Fig. 2 Unbalanced exchange flow. The variation of dimensionless volume flow rate exchanged $\widehat{Q}_{e x}$ against dimensionless displacement flow rate $\widehat{Q}_{\text {disp }}$ for a circular opening and a square opening. The lines intersect at $\widehat{Q}_{\text {disp }}=0.088$ (to 2 s.f.). There is unbalanced exchange flow whenever $\widehat{Q}_{e x}>0$ and $\widehat{Q}_{d i s p}>0$. There is displacement flow when $\widehat{Q}_{\text {disp }}>0.29$ for circular openings and $\widehat{Q}_{\text {disp }}>0.22$ for square openings. If $\widehat{Q}_{\text {disp }}=0$ then there is balanced exchange flow

It is clear from Fig. 2 that unbalanced exchange flow removes the theoretical contradiction between displacement flow theory and balanced exchange flow theory. As $a_{s} \rightarrow 0$, $\widehat{Q}_{\text {disp }} \rightarrow 0$, but as it does so there must be a point where $\widehat{Q}_{\text {disp }}=\widehat{Q}_{C}$, after which the flow will no longer be a displacement flow but unbalanced exchange flow. The total flow rate of buoyant air out of the primary opening $\widehat{Q}_{d i s p}+\widehat{Q}_{e x}$ will vary smoothly and monotonically as $a_{s}$ is reduced until, with $a_{s}=0$, there is only exchange flow-the balanced exchange limit.

Under a night cooling scenario, it is not obvious what the effect of unbalanced exchange flow will be on emptying times. Will there be a finite emptying time as with displacement flow (13) or will the room never fully empty as with balanced exchange flow (17) and perfect mixing? We will investigate this next.

\section{Effect of unbalanced exchange on displacement flows}

An important consequence of the existence of the critical flow rate, $\widehat{Q}_{C}$, can be deduced from (14). Regardless of the initial flow rate $\widehat{Q}_{\text {disp }}(\hat{t}=0)$ at which the night purge commences, $\hat{Q}_{\text {disp }}(\hat{t})$ will decrease linearly with time towards zero as the buoyancy force driving the ventilation is ever diminishing (as buoyancy is lost continuously through the primary opening). However, this means that for some $0<\widehat{t}_{C}<1$, where $\widehat{t}_{C}=t_{C} / t_{\text {disp }}$, $\widehat{Q}_{\text {disp }}\left(\hat{t}_{C}\right)=\widehat{Q}_{C}$ and unbalanced exchange flow will initiate at the primary opening. Thus for $t>t_{C}$, modelling the purge as a displacement flow is no longer valid. Assuming the initial flow rate $\widehat{Q}_{\text {disp }}>\widehat{Q}_{C}$, i.e. night cooling commences with displacement flow, substituting $\widehat{Q}_{\text {disp }}=\widehat{Q}_{C}$ into (12) and (13) gives the critical layer depth $\widehat{h}_{C}$ and time $\widehat{t}_{C}$ at which unbalanced exchange initiates, namely,

$$
\frac{h_{C}}{H}=\widehat{h}_{C}=\frac{\phi}{2}\left(1+\frac{1}{R^{2}}\right) \widehat{Q}_{C}^{2}
$$

and 


$$
\frac{t_{C}}{t_{\text {disp }}}=\widehat{t}_{C}=1-\left(\frac{\phi}{2}\right)^{1 / 2}\left(1+\frac{1}{R^{2}}\right)^{1 / 2} \widehat{Q}_{C},
$$

respectively.

If we consider our example room with $\phi=1 / 3, R=1$ and $\widehat{Q}_{C}=0.22$ then $t_{C} / t_{\text {disp }}=0.87$ and $h_{C} / H=0.016$, (each to 2 s.f.). This suggests that the fact that displacement flows will transition to unbalanced exchange flows during night cooling is not significant; for this example case the flow does not transition until $\sim 90 \%$ of the (idealised) emptying time, at which instant the only buoyancy remaining is contained within a layer whose depth is $\sim 1 \%$ of the room height. With such a thin layer, other assumptions that underpin the theory of displacement flow, such as that the pressures are hydrostatic, have already broken down and other dynamical effects come into play, for example selective withdrawal $[2,26]$.

It is worth considering whether it would be possible to avoid the onset of exchange by manipulating the opening geometry such that once $\widehat{Q}_{\text {disp }}=\widehat{Q}_{C}$, the volume flow rate does not decrease further, but still enable the purge to complete. From (24) we see that this is not possible. The only way to achieve $\widehat{h}_{C}=0$ is with $\phi=0$, which can only be achieved if $a_{p} \equiv 0$, so that there would then be zero volume flow rate.

For other room and opening geometries, the effect of unbalanced exchange flow may be significant, and it is not obvious if there will be a finite emptying time or an infinite decay of the buoyancy within the room once unbalanced exchange initiates. To answer this question we proceed by developing a mathematical model for the purge of a room which incorporates the possibility of unbalanced exchange flow.

In order to model the evolution of the layer depth and buoyancy $\left(\hat{h}\right.$ and $\left.\hat{g}^{\prime}\right)$ for $\hat{t}>\hat{t}_{C}$, the extent to which the incoming air mixes with the buoyant layer needs to be considered. As discussed in Sect. 4, for a sufficiently thin layer (i.e. $\widehat{h} \ll 1)$, the temperature of the (cool) incoming air will have increased only marginally towards the layer temperature on reaching the bottom of the layer, so the simplifying assumption of no mixing is most reasonable in this case. By contrast, for sufficiently deep layers the temperature of the descending air at the base of the layer is closer to that of the layer than the ambient and the simplifying assumption that this air is equal in temperature to the layer is not unreasonable, i.e. there is 'perfect' mixing. In reality, the true extent of the mixing will lie in between perfect and no mixing, however there are too many unknowns to model this currently. Instead, we will model unbalanced exchange flow with both mixing assumptions, placing bounds on the true behaviour.

Regardless of the form of the assumed mixing, for $0<\hat{t}<\hat{t}_{C}$ there is no unbalanced exchange, so $\hat{h}$ is described by (12) and $\hat{g}^{\prime}=1$. At $\hat{t}=\hat{t}_{C}$, (12) is no longer valid and the mixing of ambient air into the buoyant layer will reduce $\hat{g}^{\prime}$. We first consider perfect mixing, whereby incoming air is assumed to instantaneously mix with the buoyant layer, thereby decreasing its buoyancy.

\subsection{Perfect mixing}

Conservation of buoyancy and volume for the buoyant layer yield the following coupled equations for the time variation of the buoyancy, $\widehat{g}^{\prime}$, and layer depth, $\widehat{h}$ 


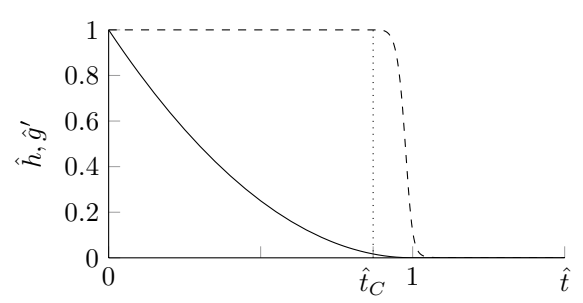

(a) $R=1$.

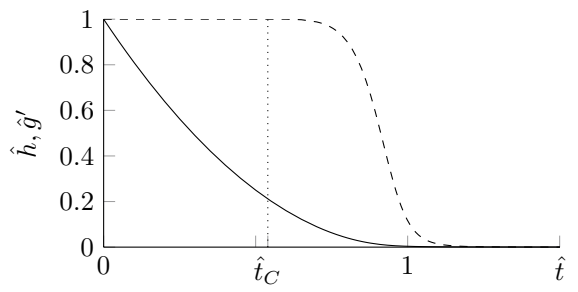

(c) $R=1 / 5$.

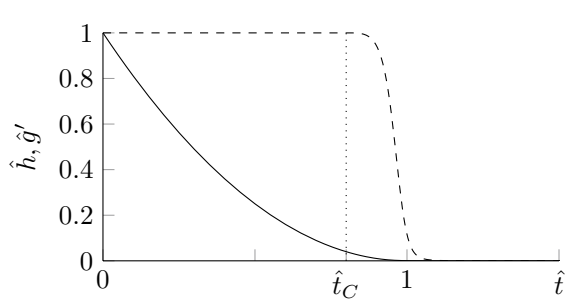

(b) $R=1 / 2$.

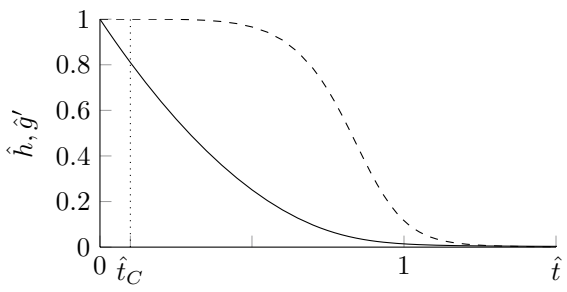

(d) $R=1 / 10$.

Fig. 3 The variation of $\hat{h}$ (solid line) and $\hat{g}^{\prime}$ (dashed line) with time $\hat{t}$ for decreasing values of $R=a_{s} / a_{p}$ (a-d). $\phi=1 / 3$. The critical times $\hat{t}_{C}$ at which unbalanced exchange commences are indicated with a dotted line. The values of $\hat{t}_{C}=0.87,0.80,0.54$ and 0.10 (to 2 s.f.) for (a-d) respectively. Note the increasingly earlier onset of unbalanced exchange flow (lower $\hat{t}_{C}$ ) as $R$ decreases

$$
\left.\begin{array}{l}
\frac{d \hat{g}^{\prime}}{d \widehat{t}}=-(2 \phi)^{1 / 2}\left(1+\frac{1}{R^{2}}\right)^{1 / 2} \frac{\hat{g}^{\prime 3 / 2}}{\widehat{h}} \widehat{Q}_{e x}\left(\widehat{Q}_{\text {disp }}\right) \\
\frac{d \hat{h}}{d \hat{t}}=-2(\widehat{g} \hat{h})^{\prime 1 / 2}
\end{array}\right\} \text { for } \hat{t}>\hat{t}_{C}
$$

where

$$
\widehat{Q}_{\text {disp }}=\left(\frac{2 \hat{h}}{\phi}\right)^{1 / 2}\left(1+\frac{1}{R^{2}}\right)^{-1 / 2}
$$

and $\widehat{Q}_{e x}\left(\widehat{Q}_{\text {disp }}\right)$ is the appropriate function for either a circular (22) or square opening (23). The system of Eq. (26) is to be solved subject to the conditions at the onset of the unbalanced exchange, namely,

$$
\widehat{h}=\widehat{h}_{C} \quad \text { and } \quad \hat{g}^{\prime}=1 \quad \text { at } \quad \hat{t}=\widehat{t}_{C} .
$$

With reference to (7), we note that $\hat{t}=1$ is the dimensionless time it would take for a room to empty of buoyant fluid under displacement flow if the flow never transitioned to unbalanced exchange flow, though we know now this can not be the case. Equations (26) and (27) are solved numerically using the Runge-Kutta method (ode45 in MATLAB).

The variation of $\hat{g}^{\prime}$ and $\hat{h}$ with time are plotted in Fig. 3 for various values of $R$ and $\phi=1 / 3$. If there were no unbalanced exchange flow, equivalent to $\hat{Q}_{C}=0$, all plots in Fig. 3 would be identical, with $\hat{h}=0$ at $\hat{t}=1$ and no variation in $\hat{g}^{\prime}$ for $\hat{t} \in[0,1]$. With the inclusion of unbalanced exchange, once $\hat{t}=\widehat{t}_{C}, \widehat{Q}_{e x} \neq 0$ and $\hat{g}^{\prime}$ decreases for $\hat{t}>\hat{t}_{C}$. This has the effect of decreasing the rate at which the buoyant layer is purged and, therefore, of 


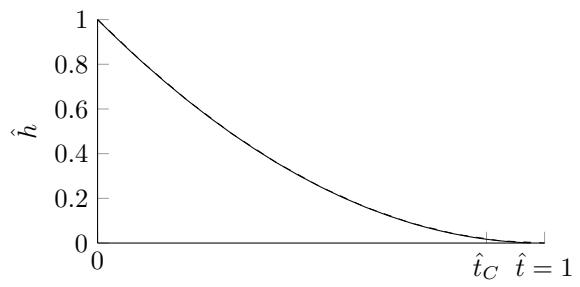

(a) $R=1$.

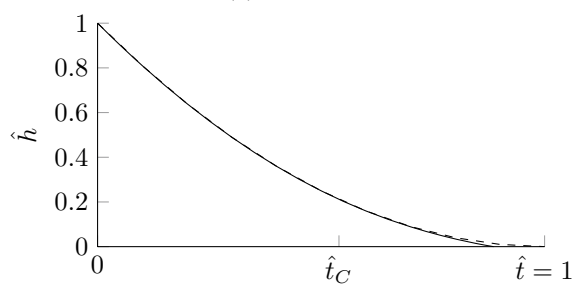

(c) $R=1 / 5$.

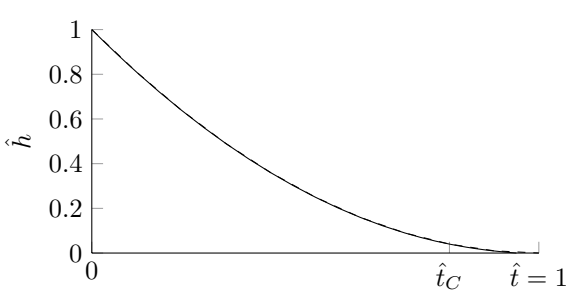

(b) $R=1 / 2$.

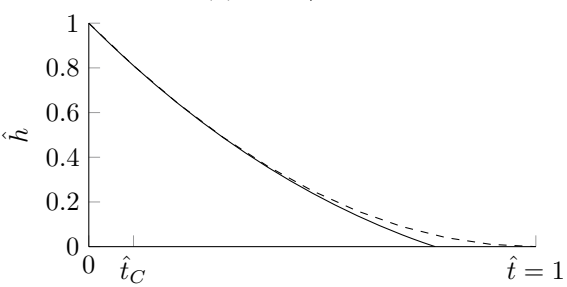

(d) $R=1 / 10$.

Fig. 4 The variation of $\hat{h}$ with time $\hat{t}$ for decreasing values of $R=a_{s} / a_{p}(\mathrm{a}-\mathrm{d}) . \phi=1 / 3$. The critical time $\hat{t}_{C}$ at which unbalanced exchange initiates is marked. The dashed line shows the trend, from (12), if there were only displacement flow. The commencement of exchange flow for $\hat{t}>\hat{t}_{C}$ always increases the total flow rate, therefore the buoyant layer is purged faster and $\widehat{t}_{E}<1 \forall R>0$

increasing the time taken for the room to fully purge. It can be seen from Fig. 3 though, that this increase is relatively small, even for $R=1 / 10$.

For this reason it seems reasonable to neglect the effect of unbalanced exchange when calculating the emptying time, $t_{E}$, if we assume that there is perfect mixing between the incoming unbalanced exchange flow and the buoyant layer. If it is important that there is no inflow through the top (i.e. primary) opening however, perhaps for reasons of external air quality, then it would be wise to select primary and secondary openings so that $R=a_{s} / a_{p}$ is as large as possible, thus minimising the proportion of the total emptying time where unbalanced exchange would be expected. Moreover, based on the results of Hunt and Coffey [10], this would also serve to mitigate interfacial mixing due to the inflow through the secondary opening.

\subsection{No mixing}

We now consider the other extreme, namely, of no mixing between the inflow through the primary opening and the buoyant layer. As before, the problem is already solved for $0<\hat{t}<\hat{t}_{C}$ and, thus, we only need to solve for $\hat{t}>\hat{t}_{C}$. In the absence of mixing there is no change of the buoyancy of the layer and therefore $\widehat{g}^{\prime}=1$ for $\hat{t}>\widehat{t}_{C}$. Conservation of volume for the buoyant layer then gives

$$
\frac{d \widehat{h}}{d \widehat{t}}=-2 \widehat{h}^{1 / 2}-(2 \phi)^{1 / 2}\left(1+\frac{1}{R^{2}}\right)^{1 / 2} \widehat{Q}_{e x}\left(\widehat{Q}_{\text {disp }}\right), \quad \text { for } \hat{t}>\widehat{t}_{C}
$$

with $\widehat{Q}_{e x}$ and $\widehat{Q}_{\text {disp }}$ defined as before, and which must be solved subject to 


$$
\hat{h}=\hat{h}_{C} \quad \text { at } \quad \hat{t}=\hat{t}_{C}
$$

where $h_{C}$ and $t_{C}$ are given in (24) and (25), respectively. The second term on the right-hand side of (29) represents the change in layer depth due to exchange flow. This term is negative because, in the absence of mixing, the exchange flow coming in the primary opening passes through the buoyant layer without interacting with it. This has the effect of increasing the flow rate, thereby purging the buoyant layer faster. The variation of $\hat{h}$ with time for four values of $R$ are plotted in Fig. 4. In contrast to the predictions based on perfect mixing, with no mixing there is a finite draining time of $\hat{t}<1$ for all values of $R$. This is because, with no mixing, $\hat{g}^{\prime}$ is constant throughout the draining process, so the total flow rate is always greater than or equal to the flow rate with displacement flow alone, for which there is a finite draining time.

Given unbalanced exchange flow initiates typically when $\hat{h} \ll 1$, then $\widehat{Q}_{\text {disp }}=\widehat{Q}_{s} \ll 1$ and, hence the exchange is almost balanced $\widehat{Q}_{e x} \approx \widehat{Q}_{b a l}$. Moreover, from (22) and (23), $\widehat{Q}_{e x}=$ constant to leading order. Thus, Eq. (29) may be approximated as

$$
\frac{d \hat{h}}{d \hat{t}}=-2 \widehat{h}^{1 / 2}-c
$$

and $c=2^{1 / 2}\left(1+R^{-2}\right)^{1 / 2} \phi^{1 / 2} \widehat{Q}_{b a l}$. The initial condition is $\widehat{h}=\widehat{h}_{C}$ because (31) is only valid once unbalanced exchange initiates. The ordinary differential Eq. (31) has the following analytical solution

$$
\hat{h}=\frac{c^{2}}{4}(W(X)+1)^{2} \quad \text { where } \quad X=\frac{\left(-c-2 \hat{h}_{C}^{1 / 2}\right)}{c} \exp \left(\frac{2 \widehat{t}-c-2 \widehat{h}_{C}^{1 / 2}}{c}\right)
$$

and $W(X)$ is the Lambert $W$-function [19]. Setting $\widehat{h}=0$ in (32) and rearranging for $\hat{t}$ gives the time, $\Delta \widehat{t}$, for $\widehat{h}$ to reduce from $\widehat{h}_{C}$ to 0 as

$$
\Delta \widehat{t}=\widehat{h}_{C}^{1 / 2}-\frac{c}{2} \ln \left(1+\frac{2 \widehat{h}_{C}^{1 / 2}}{c}\right) .
$$

The total emptying time is therefore $\widehat{t}_{E}=\widehat{t}_{C}+\Delta \widehat{t}$. Noting that

$$
\widehat{t}_{C}=1-\widehat{h}_{C}^{1 / 2} \quad \text { and } \quad \frac{2 \hat{h}_{C}^{1 / 2}}{c}=\frac{\widehat{Q}_{C}}{\widehat{Q}_{b a l}},
$$

we find that the emptying time, on accounting for both the displacement flow phase and the subsequent unbalanced exchange flow phase, may be approximated as

$$
\hat{t}_{E}=1-\underbrace{\left(\frac{\phi}{2}\right)^{1 / 2}\left(1+\frac{1}{R^{2}}\right)^{1 / 2}}_{\text {Room geometry }} \underbrace{\hat{Q}_{b a l} \ln \left(1+\frac{\widehat{Q}_{C}}{\hat{Q}_{b a l}}\right)}_{\text {Opening shape }} .
$$

From (35) we see that the effect of unbalanced exchange, if no mixing is assumed, is to reduce the emptying time for all room and opening geometries. Note that the emptying time is reduced by increasing $\phi$ or decreasing $R$ (the terms labelled 'Room geometry'), and also depends on the shape of the primary opening (the terms labelled 'Opening shape'). It 


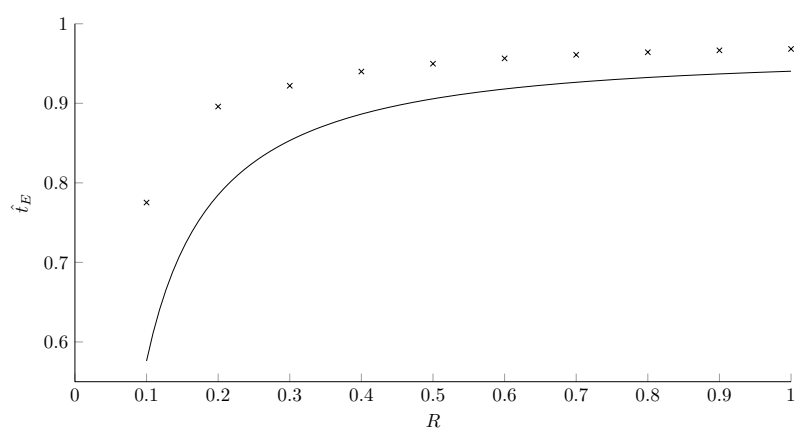

Fig. 5 The variation of emptying time, $\widehat{t}_{E}$, with opening area ratio $R$ in the absence of mixing. $\phi=1 / 3$ and square openings are modelled so that $\widehat{Q}_{C}=0.22$ and $\widehat{Q}_{b a l}=0.076$ (the latter value obtained by setting $\widehat{Q}_{\text {disp }}=0$ in (23)). The solid line shows the analytical approximation to the emptying time (35). The crosses show values calculated using (29). Equation (35) always underestimates the emptying time, however the error decreases as $R$ increases

should also be noted that (35) is only valid for $\hat{h}_{C} \ll 1$; if $\hat{h}_{C}$ is made large by increasing $\phi$ or reducing $R$ then $\hat{t}_{E}$ could be made negative, which is clearly nonsensical. The variation of calculated and approximate emptying time with $R$ and $\phi$ is plotted in Fig. 5.

For the example room considered, namely, with $\phi=1 / 3$ and $R=1$ and square openings so that $\widehat{Q}_{b a l}=0.076$ and $\widehat{Q}_{C}=0.22$, (35) gives the approximation $\widehat{t}_{E}=0.94$ (to 2 s.f.), compared to $\hat{t}_{E}=0.97$ calculated numerically. We expect (35) to underestimate $\widehat{t}_{E}$ because it assumes $\widehat{Q}_{e x}=\widehat{Q}_{b a l}$ which is only true when $\widehat{Q}_{s}=0$. In general $\widehat{Q}_{e x} \leq \widehat{Q}_{b a l}$, however (35) provides a useful first-order estimate and highlights the dependence of the emptying time on the controlling dimensionless parameters.

One aspect of interest that is not possible to investigate using the analysis in this section is how the introduction of a low-level secondary opening impacts balanced exchange flow. The timescale used is the emptying time with displacement flow alone, $t_{d i s p}$, which with a balanced exchange flow is infinite, meaning the equations break down. In order to compare balanced with unbalanced exchange flow, we need a new timescale.

\section{The benefits of unbalanced over balanced exchange flow}

If we are to compare balanced with unbalanced exchange flows, the timescale must be meaningful for all values of $R$, including $R=0$. Accordingly, we introduce the new timescale

$$
t^{*}=\frac{2 S}{a_{p}}\left(\frac{H^{\prime}}{g_{0}}\right)^{1 / 2}=2^{1 / 2}\left(1+\frac{1}{R^{2}}\right)^{-1 / 2} t_{d i s p}=\phi^{1 / 2} \widehat{Q}_{b a l} t_{b a l}
$$

so that the corresponding non-dimensional time is

$$
\hat{t}^{*}=t / t^{*}
$$


Based on this definition, a room with $R=1$ would evidently empty in a time $t_{\text {disp }}$ if the displacement flow that commences at $t=0$ never transitioned to unbalanced exchange. However, as $t^{*}$ is independent of $R$, non-dimensionalising the governing equations by $t^{*}$ means they are valid for $R=0$ as well as other values of $R$ and, therefore, this choice of scaling allows for the comparison of balanced with unbalanced exchange. All other scalings are unchanged for the purposes of the comparison.

As in Sect. 7, we first consider the case of perfect mixing. In Sect. 7.1 we calculated the time evolution of buoyancy $\left(g^{\prime}\right)$ and layer depth $(h)$ separately, but the purpose of night cooling is to remove excess heat, i.e. the buoyancy, regardless of whether this is achieved by reducing $g^{\prime}$ or $h$. Accordingly, we consider the total buoyancy in the space $B(t)$, a product of $g^{\prime}$ and $h$, given by integrating the buoyancy over the room volume $V$ :

$$
B(t)=\int_{V} g^{\prime} d V .
$$

As we assume 'perfect' mixing, $g^{\prime}$ is uniformly distributed throughout the layer, which occupies the full height of the room, so the integral in (38) may be evaluated straightforwardly to give $B(t)=S H g^{\prime}(t)$. Normalising the instantaneous value of $B(t)$ by the total buoyancy in the room at time $t=0$, namely, $B_{0}=S H g_{0}^{\prime}$, we define

$$
\widehat{B}=B / B_{0} .
$$

Based on these new scalings, the governing equations become

$$
\left.\begin{array}{l}
\frac{d \widehat{B}}{d \widehat{t}^{*}}=-2^{3 / 2}\left(1+\frac{1}{R^{2}}\right)^{-1 / 2} \frac{\widehat{B}^{3 / 2}}{\widehat{h}} \\
\frac{d \widehat{h}^{2}}{d \widehat{t}^{*}}=-2^{3 / 2}\left(1+\frac{1}{R^{2}}\right)^{-1 / 2} \widehat{B}^{1 / 2},
\end{array}\right\} \text { for } 0<\widehat{t}^{*}<\widehat{t}_{C}^{*}
$$

subject to the initial conditions

$$
\widehat{B}=1 \quad \text { and } \quad \hat{h}=1 \quad \text { at } \quad \widehat{t}^{*}=0
$$

and, cf. (26),

$$
\left.\begin{array}{l}
\frac{d \widehat{B}}{d \widehat{t}^{*}}=-2^{3 / 2}\left(1+\frac{1}{R^{2}}\right)^{-1 / 2} \frac{\widehat{B}^{3 / 2}}{\widehat{h}}-2 \phi^{1 / 2} \frac{\widehat{B}^{3 / 2}}{\widehat{h}^{3 / 2}} \widehat{Q}_{e x}\left(\widehat{Q}_{\text {disp }}\right) \\
\frac{d \hat{h}}{d \widehat{t}^{*}}=-2^{3 / 2}\left(1+\frac{1}{R^{2}}\right)^{-1 / 2} \widehat{B}^{1 / 2},
\end{array}\right\} \text { for } \hat{t}^{*}>\widehat{t}_{C}^{*}
$$

with $\widehat{Q}_{\text {disp }}$ and $\widehat{Q}_{e x}$ defined as before. The solution of the coupled Eq. (42) is required subject to the initial conditions

$$
\widehat{B}=\widehat{h}=\widehat{h}_{C} \quad \text { at } \quad \hat{t}^{*}=\widehat{t}_{C}^{*}
$$

The variation of $\widehat{B}$ with time $\hat{t}^{*}$ in a room with square openings, assuming perfect mixing, is plotted in Fig. 6a for values of $R$ ranging from 0 to 1 , with $\phi=1 / 3$.

If we were instead to assume no mixing, because there is then no change in $\hat{g}^{\prime}$, the change in $\widehat{B}$ is simply the change in $\hat{h}$. Therefore we need only change the timescale in (29) to (36) to give 
Fig. 6 The variation of nondimensionalised buoyancy $\widehat{B}$ with time $\hat{t}^{*}$ for different values of $R$ (see Legend) in a room with square openings and $\phi=1 / 3$ assuming a perfect mixing and $\mathbf{b}$ no mixing. The timescale is chosen so that with $R=1$ the room would be empty in time $\hat{t}^{*}=1$ if there were no unbalanced exchange. With unbalanced exchange and perfect mixing the room with $R=1$ is $99 \%$ empty in $\hat{t}_{99}^{*}=0.90$ but never completely empty. With unbalanced exchange and no mixing the room with $R=1$ is empty in time $\hat{t}_{E}^{*}=0.97$

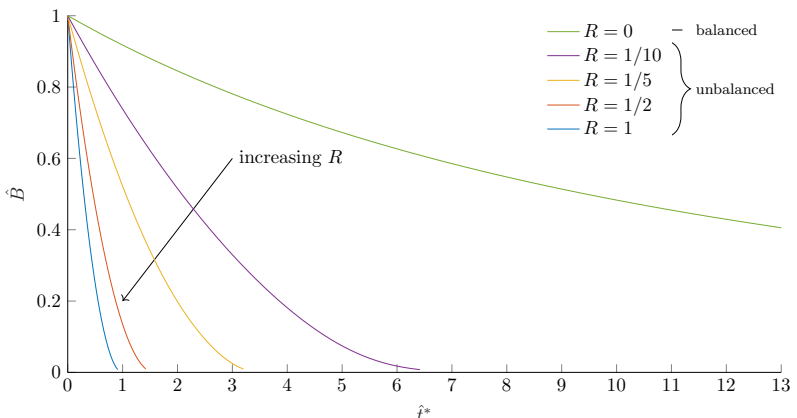

(a) Perfect mixing

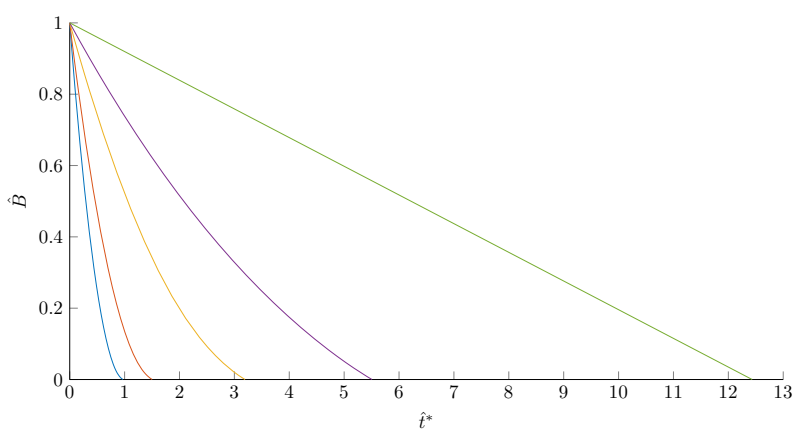

(b) No mixing

$$
\left.\frac{d \widehat{B}}{d \widehat{t}^{*}}=-2^{3 / 2}\left(1+\frac{1}{R^{2}}\right)^{-1 / 2} \widehat{B}^{1 / 2}-2 \phi^{1 / 2} \widehat{Q}_{e x}\left(\widehat{Q}_{\text {disp }}\right) \quad\right\} \text { for } \widehat{t}^{*}>\widehat{t}_{C}^{*} .
$$

Solutions to (44), subject to $\widehat{B}=\widehat{B}_{C}=\widehat{h}_{C}$ at $\widehat{t}^{*}=\widehat{t}_{C}^{*}$, are plotted in Fig. $6 \mathrm{~b}$ for a room with square openings. For $\hat{t}^{*}<\hat{t}_{C}^{*}$ the solutions to (40) are plotted.

Figures $6 \mathrm{a}$ and $\mathrm{b}$ clearly show the benefit of introducing even a relatively small lowlevel secondary opening (e.g. as achieved on increasing $R$ from zero to $R=1 / 10$ ) over none at all $(R=0)$. With perfect mixing, when $R=0$ (no low-level opening) it takes $\hat{t}^{*}>9$ for half of the total initial buoyancy to be purged from the room, whereas with $R=1 / 10$, i.e. a secondary opening one tenth of the area of the primary opening, the room is $50 \%$ purged after approximately $\widehat{t}^{*}=2$. With no mixing, moving from $R=0$ to $R=1 / 10$ reduces the emptying time by more than half.

By comparing Fig. $6 \mathrm{a}$ and $\mathrm{b}$ we can also see that the choice of mixing assumption is not important practically for most values of $R$; only with $R \leq 0.1$ do the times taken to purge the bulk of the initial buoyancy differ by more than $10 \%$. As the assumption of perfect mixing is more appropriate for $R \leq 0.1$ and the mixing assumption does not make a significant difference to the emptying times for $R>0.1$, it is reasonable to assume perfect mixing for unbalanced exchange flow calculations. This also has the benefit of being the conservative approach, which is best practice for ventilation calculations, etc.

Despite having a non-zero displacement flow component, unbalanced exchange flows share a similarity with balanced exchange flow in that the room never purges fully. Indeed, in Fig. 6a and b, $\widehat{B}$ asymptotes to $\widehat{B}=0$ in the limit as $\hat{t}^{*} \rightarrow \infty$. Thus, in order to quantify the difference in effectiveness of purging for different geometries we define $\widehat{t}_{99}^{*}$ as the time taken for $99 \%$ of the buoyancy to be purged from the room, i.e. 
Fig. 7 The variation of $\hat{t}_{99}^{*}$, the time taken for $99 \%$ of the initial total buoyancy to be purged from a room, with $R=a_{s} / a_{p}$ and $\phi=1 / 3,1 / 6$ and $1 / 12$, assuming perfect mixing
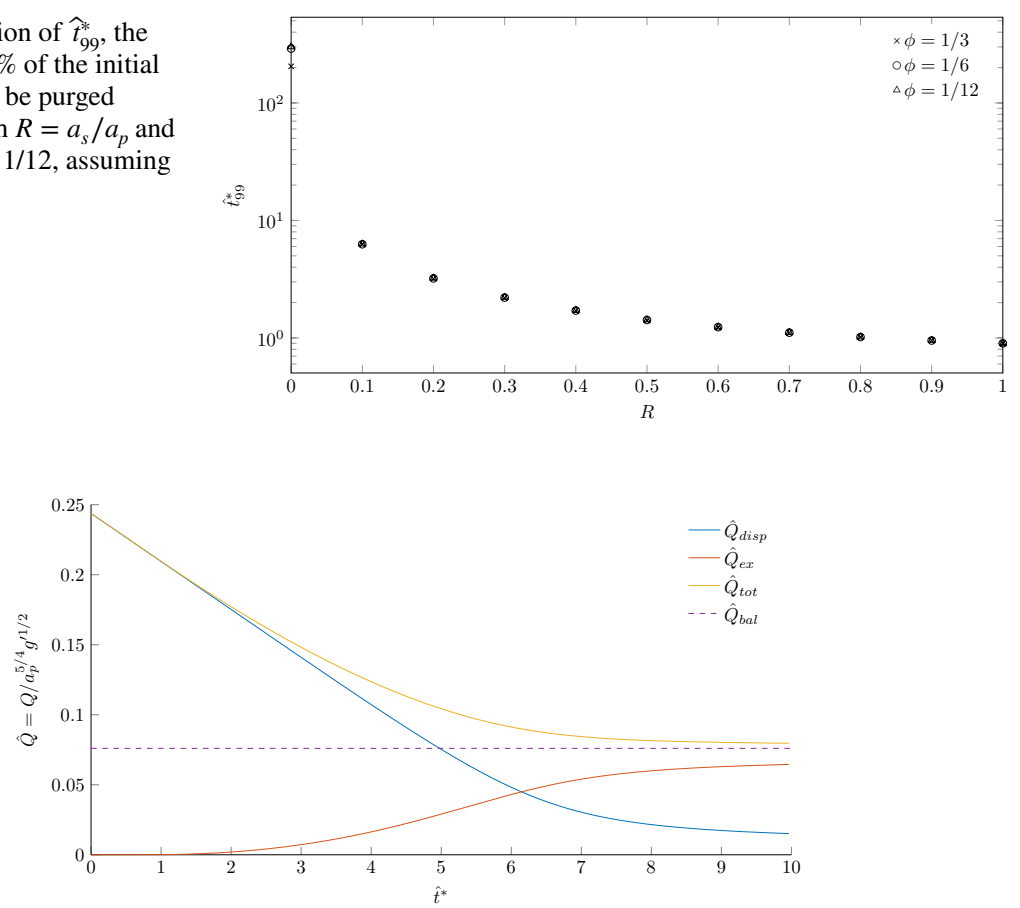

Fig. 8 A comparison of volume flow rates over the duration of a night purge. The solid lines are flow rates for $R=1 / 10$ and the dashed line for $R=0$, i.e. balanced exchange flow. The initial displacement flow rate $\widehat{Q}_{\text {disp }}$ is three times greater than the balanced exchange flow rate $\widehat{Q}_{b a l}$ and the total flow rate $\widehat{Q}_{t o t}=\widehat{Q}_{\text {disp }}+\widehat{Q}_{e x}$ is always greater than $\widehat{Q}_{b a l}$; this explains why the addition of a small low-level secondary opening enhances the rate of removal of buoyancy from a room

$\widehat{B}\left(\hat{t}^{*}=\widehat{t}_{99}^{*}\right)=0.01$. The variation of $\hat{t}_{99}^{*}$ with $R$ is plotted in Fig. 7 for the case of perfect mixing, for $\phi=1 / 3,1 / 6$ and $1 / 12$. Only with $R=0$ is there a graphically distinguishable difference between the values, because although (42) does depend on $\phi$, this is only in the exchange term, not the displacement term. The displacement term dominates the exchange term (see Fig. 8) and so only with balanced exchange, i.e. $R=0$, does $\phi$ affect $\hat{t}_{99}^{*}$.

From Fig. 7 we can see that with $R=1 / 10, \hat{t}_{99}^{*}=6.3$ (to 2 s.f.), whereas with $R=0$, i.e. balanced exchange $\hat{t}^{*}=205$. The reason for this dramatic difference is the large magnitude of the displacement flow rate component relative to the exchange flow rate component, even at small values of $R>0$. The relative magnitudes of the volume flow rates achieved during the night cooling example plotted in Fig. 6 are shown in Fig. 8. For $R=1 / 10$ both the separate components $\widehat{Q}_{\text {disp }}$ and $\widehat{Q}_{e x}$, and their sum $\widehat{Q}_{t o t}=\widehat{Q}_{e x}+\widehat{Q}_{e x}$ are plotted.

With $R=0$, the balanced exchange flow case, $\widehat{Q}_{\text {disp }} \equiv 0$ and $\widehat{Q}_{e x}=\widehat{Q}_{b q l}=0.076$ for the entire duration of the purge. However, with $R=1 / 10$, the initial value of $Q_{\text {disp }}=0.24$, over three times greater than $\widehat{Q}_{e x}$ during balanced exchange. Although with $R=1 / 10$ unbalanced exchange initiates relatively early during the purge, at around $\hat{t}^{*}=1$, the exchange flow component $\widehat{Q}_{e x}$ only exceeds the displacement flow component $\widehat{Q}_{\text {disp }}$ for $\hat{t}^{*}>6$, by which time it can be seen in Fig. 6 that the room is almost empty of buoyant fluid. Displacement flows, and the displacement flow component of unbalanced exchange flows, are 
far more effective at purging buoyancy from a space than balanced exchange flows, and the exchange flow component of unbalanced exchange flows. As a consequence, a low-level opening should be provided for natural ventilation schemes wherever possible, even if it is much smaller in area than the high-level openings.

\section{Discussion}

The three component parts that underpin and occur sequentially in our analysis without interaction, namely, displacement flow theory, the transition to unbalanced exchange flow and the theory of unbalanced exchange flow have each been validated previously. The validation we refer to is based on controlled laboratory experiments in which fresh and salt water are used as the working fluids in order to achieve approximate dynamical similarity in small-scale models of a room [6, 7, 10, 16, 23]. It is this validation that gives us confidence in our analysis. First, we have established that for the majority of the purging time there will be displacement flow, the theory for which has been extensively validated, for example, by Linden, Lane-Serff and Smeed [16] and Hunt and Coffey [10], amongst others. Second, the value we use for $\widehat{Q}_{C}$, the critical flow rate for transition to unbalanced exchange, is based on the independent measurements of Epstein and Kenton [7] and Hunt and Coffey [10], and explained theoretically by Wise and Hunt [25]. Third, as detailed in [25], the theory of unbalanced exchange flow [25] has been validated by comparison with the measurements of both Epstein and Kenton [7] and Varrall, Pretrel, Vaux and Vauquelin [23].

Whilst this validation provides confidence in our analysis, a more detailed validation would ideally require the use of scale models that are far larger than those used previously-previous models having a vertical dimension on the order of $1 \mathrm{~m}$-and achieving even approximate dynamical similarity for the entire duration of the purge will likely present a non-trivial challenge. These comments stem from the fact that the buoyant layer is thin when transition occurs (Sect. 7) meaning that the velocities through the primary opening will be relatively small and the flow may not be independent of Reynolds number, either at model or full scale. This, in turn, begs the question of what form should the loss coefficients $\left(c_{s}, c_{p}\right)$ then take; the 'loss' coefficient conventionally accounting both for frictional losses due to fluid contact with the opening and the contraction of the flow through the opening. As our focus herein has been to better understand the fundamentals of purging, we intentionally neglected these effects, in effect taking each coefficient to be equal to unity $^{2}$ Whilst these coefficients are well defined for unidirectional flow through an opening [8], it is not clear what is meant by a loss coefficient for an opening with bi-directional flow, such as balanced or unbalanced exchange flow. As a consequence, we anticipate that comparisons of experimental data to the predictions of the theory may differ during the late stage transients, not as a consequence of the underlying fundamental theory but because of a lack of understanding of loss coefficients. Nonetheless, the theory developed herein is

\footnotetext{
${ }^{2}$ The framework of our analysis readily allows for loss coefficients to be introduced explicitly and their role examined simply by redefining $R$ in (4) to be the ratio of the effective, rather than physical, areas so that $R=c_{s} a_{s} /\left(c_{p} a_{p}\right)$. For first-order predictive purposes, in line with much of the building ventilation literature it would not appear unreasonable to take $c_{p}=c_{s}=0.6$ but to be aware that these coefficients will vary with Reynolds number [24] during the final stages of the purge and that for unidirectional outflow $c_{p}$ has been shown to vary with the Richardson number of the outflow [9].
} 
useful to explain trends which we would expect to be observed in experiments and to carry over into full-scale ventilation systems.

\section{Conclusions}

A mathematical model for the buoyancy-driven self-purging of fluid from a box-shaped room via upper and lower openings that connect to denser surroundings has been developed with the primary focus and application being to the night purging of heat from a building as a low-energy sustainable solution. Our theoretical investigation has thrown new light on the complex nature of such a night purge and elucidated how a key dynamical feature, in the form of a flow transition, has been overlooked until now. We show that this transition fundamentally alters the rate of cooling from that of a displacement flow and the time taken to complete a purge. Our focus throughout has been to investigate how the passive flow transports heat out of a room (of height $H(\mathrm{~m})$ with floor area $S\left(\mathrm{~m}^{2}\right)$ ) and hence all boundaries have been assumed to be insulating.

Prior to this investigation it has been widely accepted that a displacement flow established at the start of a night purge is maintained for the entire duration of the purge, i.e. until all warm air has been displaced from the room. Consequently, the emptying time $t_{E}=t_{d i s p}$, (13), has been routinely estimated by practitioners based on idealised displacement flow theory (Sect. 3), wherein the inflow of cool night air through the secondary opening and the outflow of warm air through the primary opening are assumed to be maintained throughout.

Displacement flow is not maintained however, and we have established herein how the emptying time, a quantity essential for effective passive ventilation design, differs from that predicted by the idealised theory. During the purge, the flow must transition from displacement flow to 'unbalanced exchange flow' in which there is simultaneously inflow of cool air and outflow of warm air at the top opening. Only the inflow of cool night air through the base opening is maintained. As such, we deduce that the effective opening area is not the key quantity that characterises the openings, instead openings for a night purge are characterised by the ratio of opening areas $R=a_{s} / a_{p}$ and the scale of the primary opening relative to the room height $\phi=\sqrt{a_{p}} / H$. Moreover, we establish that the form of the mixing between cool air flowing in at the top and the air within the room plays a key role in the predicted behaviour of the purge, behaviours we bound by considering the extremes of perfect mixing and no mixing.

The theoretical model we develop predicts the duration over which displacement flow is maintained prior to the onset of unbalanced exchange, and the emptying time taking into account the flow transition. This emptying time departs further and further from that predicted by the idealised theory as the ratio $R$ decreases. Our model regards the unbalanced exchange flow as being comprised of a displacement flow component and an exchange flow component. For a typical room we show that the magnitude of the difference in emptying time is circa $\sim 10 \%$ when the exchange flow component is relatively weak (for $R>1 / 10$ ), regardless of the mixing assumption. In other words, estimating the emptying time from idealised displacement flow theory is not unreasonable for $R>1 / 10$. For $R<1 / 10$ the exchange flow component is relatively strong and we provide an analytical solution (35) for the emptying time which clearly reveals the role that the unbalanced exchange flow has on the purge. 
Finally, for rooms designed to purge with a balanced exchange flow strategy, i.e. with just a top opening, we have demonstrated the benefit of introducing a base opening, even if it is far smaller in area. A base opening just a tenth of the area of the top opening allows 99\% of the heat to be purged 30 times faster (approx.) than a room with just a top opening, assuming perfect mixing.

\section{Appendix}

\section{The buoyancy of a plume due to a balanced exchange flow}

In order to characterise the descending inflow from a balanced exchange flow as a plume, it is first necessary to determine whether it is forced, pure or lazy at source. In the context of the night purge considered, this source is the primary opening, which we designate as being at the coordinate origin $z=0$, with $z$ increasing downwards to base of the room at $z=H$. Given we consider here a balanced exchange flow, it is natural to make the assumption that the area of the plume source is one half of the area of the primary opening. Classical turbulent plumes can be characterised by a Richardson number which can be shown [11] to scale as

$$
\Gamma \sim \frac{\left(a_{p} / 2\right)^{5 / 2} G^{\prime}}{Q_{b a l}^{2}} \sim \widehat{Q}_{b a l}^{-2} .
$$

where $G^{\prime}=g\left(\rho_{\text {plume }}-\rho_{\text {buoy }}\right) / \rho_{\infty}$ is the buoyancy of the plume. The full expression for $\Gamma$ is

$$
\Gamma=\frac{5}{4 \alpha} \frac{\left(a_{p} / 2\right)^{5 / 2} g^{\prime}}{Q_{b a l}^{2}}=\frac{5}{2^{9 / 2} \alpha} \widehat{Q}_{b a l}^{-2},
$$

where $\alpha=0.1$ is the plume entrainment coefficient [18]. Therefore, with a square opening $\widehat{Q}_{b a l}=0.076$ and $\Gamma=380$ (to 2 s.f.), corresponding to a lazy plume at source given $\Gamma>1$. Thus, the simple geometric virtual origin correction for a pure plume [18], $z_{v}=5\left(a_{p} / 2\right)^{1 / 2} / 12 \alpha$, is not appropriate. Instead we use a virtual origin correction for highly lazy plumes $(\Gamma>88)[12]$

$$
z_{v}=0.71 \frac{\left(a_{p} / 2\right)^{1 / 2}}{\alpha \Gamma^{1 / 5}} .
$$

This gives $z_{v}=1.5 a_{p}^{1 / 2}$ (to 2 s.f.), compared with $z_{v}=2.9 a_{p}^{1 / 2}$ (to 2 s.f.) if a pure plume had been assumed. The difference in buoyancy between the air in the plume and the room air can then be calculated [18] from

$$
G^{\prime}(z)=\frac{5 F}{6 \alpha}\left(\frac{9 \alpha F}{10}\right)^{-1 / 3}\left(z+z_{v}\right)^{-5 / 3}
$$

where $F=\left.Q_{b a l} G^{\prime}\right|_{z=0}$ is the buoyancy source strength (i.e. the source buoyancy flux). There are clearly significant challenges when modelling the flow structure due to a balanced exchange flow as a plume: the source area of the plume is not well known; the plume is not steady but generated by a series of pulses [5, 22]; and it is not known whether a constant entrainment coefficient is appropriate. However, as to our knowledge no research has 
been done on the flow structure caused by a balanced exchange flow, (48) provides a useful first estimate of $G^{\prime}$ as a function of height and geometric parameters.

Substitution for $F, Q_{b a l}$ and $z_{v}$ into (48) and rearrangement in dimensionless form then gives

$$
\frac{G^{\prime}(\widehat{z})}{\left.G^{\prime}\right|_{\hat{z}=0}}=\frac{5}{6}\left(\frac{10}{9}\right)^{1 / 3} \frac{\widehat{Q}_{e x}^{2 / 3} \phi^{5 / 3}}{\alpha^{4 / 3}}(\widehat{z}+1.5 \phi)^{-5 / 3}
$$

where we recall $\phi=\sqrt{a_{p}} / H$. Taking $\alpha=0.1$ and $\widehat{Q}_{b a l}=0.076$ (for a square opening) in (49) gives the buoyancy difference at a depth $\widehat{z}$ below the primary opening as

$$
\frac{G^{\prime}(\widehat{z})}{\left.G^{\prime}\right|_{\hat{z}=0}}=\frac{3.3 \phi^{5 / 3}}{(\widehat{z}+1.5 \phi)^{5 / 3}} .
$$

However, this is clearly not self-consistent because (50) gives $G^{\prime}(0) /\left.G^{\prime}\right|_{\hat{z}=0}=3.3 / 1.5^{5 / 3}$, when we require $G^{\prime}(0) /\left.G^{\prime}\right|_{\hat{z}=0}=1$, i.e. the ambient air is unmixed as it comes through the opening. Therefore we modify (50) as

$$
\frac{G^{\prime}(\widehat{z})}{\left.G^{\prime}\right|_{\hat{z}=0}}=\frac{\beta \phi^{5 / 3}}{\left(\widehat{z}+\beta^{3 / 5} \phi\right)^{5 / 3}}
$$

and select $1.5^{5 / 3}<\beta<3.3$. Given all the approximations involved, we take $\beta=3$.

Acknowledgements The authors gratefully acknowledge the Engineering and Physical Sciences Research Council for the grant (EP/N010221/1) entitled Managing air for green inner cities (MAGIC) and for the financial support of Innovate UK (grant no. 106163 Product Based Building Solutions - High Productivity Digital Integrated Assured DFMA for Lifecycle Performance) in collaboration with Laing O’Rourke.

\section{Declarations}

Declaration The authors report no conflict of interest.

Open Access This article is licensed under a Creative Commons Attribution 4.0 International License, which permits use, sharing, adaptation, distribution and reproduction in any medium or format, as long as you give appropriate credit to the original author(s) and the source, provide a link to the Creative Commons licence, and indicate if changes were made. The images or other third party material in this article are included in the article's Creative Commons licence, unless indicated otherwise in a credit line to the material. If material is not included in the article's Creative Commons licence and your intended use is not permitted by statutory regulation or exceeds the permitted use, you will need to obtain permission directly from the copyright holder. To view a copy of this licence, visit http://creativecommons.org/licenses/by/4.0/.

\section{References}

1. Brown WG, Wilson AG, Solvason KR (1963) Heat and moisture flow through openings by convection. J Am Soc Heat Vent Air Cond Eng 5:49-54

2. Bryant PJ, Wood IR (1976) Selective withdrawal from a layered fluid. J Fluid Mech 77(3):581-591. https://doi.org/10.1017/S0022112076002267

3. CIBSE (2005) AM10 - Natural ventilation in non-domestic buildings. CIBSE

4. Coffey C, Hunt G (2007) Ventilation effectiveness measures based on heat removal: part 2. Application to natural ventilation flows. Build Environ 42(6):2249-2262. https://doi.org/10.1016/j.buildenv. 2006.03.015 
5. Conover TA, Kumar R, Kapat JS (1995) Buoyant pulsating exchange flow through a vent. J Heat Transf 117(3):641-648. https://doi.org/10.1115/1.2822625

6. Epstein M (1988) Buoyancy-driven rxchange flow through small openings in horizontal partitions. J Heat Transf 110(4a):885-893. https://doi.org/10.1115/1.3250589

7. Epstein M, Kenton MA (1989) Combined natural convection and forced flow through small openings in a horizontal partition, with special reference to flows in multicompartment enclosures. J Heat Transf 111(3):980-987. https://doi.org/10.1115/1.3250814

8. Etheridge D, Sandberg M (1996) Building ventilation?: theory and measurement. Wiley, Chichester

9. Holford JM, Hunt GR (2001) The dependence of the discharge coefficient on density contrast-experimental measurements. In: Proc. 14th Aust. Fluid Mech. Conf., Adel. Univ., vol 1014, p 123126

10. Hunt GR, Coffey CJ (2010) Emptying boxes - classifying transient natural ventilation flows. J Fluid Mech 646:137-168. https://doi.org/10.1017/S0022112009993028

11. Hunt GR, Kaye NB (2005) Lazy plumes. J Fluid Mech 533:329-338. https://doi.org/10.1017/S0022 $11200500457 \mathrm{X}$

12. Hunt GR, Kaye NG (2001) Virtual origin correction for lazy turbulent plumes. J Fluid Mech 435:377396. https://doi.org/10.1017/S0022112001003871

13. Hunt GR, Linden PF (2005) Displacement and mixing ventilation driven by opposing wind and buoyancy. J Fluid Mech 527:27-55. https://doi.org/10.1017/S0022112004002575

14. Jones BM, Cook MJ, Fitzgerald SD, Iddon CR (2016) A review of ventilation opening area terminology. Energy Build 118:249-258. https://doi.org/10.1016/j.enbuild.2016.02.053

15. Kaye NB, Hunt GR (2007) Smoke filling time for a room due to a small fire: the effect of ceiling height to floor width aspect ratio. Fire Saf J 42(5):329-339. https://doi.org/10.1016/j.firesaf.2006.12.003

16. Linden PF, Lane-Serff GF, Smeed DA (1990) Emptying filling boxes: the fluid mechanics of natural ventilation. J Fluid Mech 212:309-335. https://doi.org/10.1017/S0022112090001987

17. Lynch PM, Hunt GR (2011) The night purging of a two-storey atrium building. Build Environ 46(1):144-155. https://doi.org/10.1016/j.buildenv.2010.07.009

18. Morton BR, Taylor G, Turner JS (1956) Turbulent gravitational convection from maintained and instantaneous sources. Proc R Soc A 234(1196):1-23. https://doi.org/10.1098/rspa.1956.0011

19. Olver FWJ, of Standards and Technology (US) NI, (2010) NIST handbook of mathematical functions. Cambridge University Press, Cambridge, New York

20. Shrinivas AB, Hunt GR (2015) Confined turbulent entrainment across density interfaces. J Fluid Mech 779:116-143. https://doi.org/10.1017/jfm.2015.366

21. Taylor G (1950) The instability of liquid surfaces when accelerated in a direction perpendicular to their planes. Proc R Soc A 201(1065):192-196. https://doi.org/10.1098/rspa.1950.0052

22. Varrall K, Pretrel H, Vaux S, Vauquelin O (2016) Stereoscopic particle image velocimetry investigation of the bidirectional natural convection flow through a horizontal vent. Fire Technol 52(6):20272041. https://doi.org/10.1007/s10694-016-0593-3

23. Varrall K, Pretrel H, Vaux S, Vauquelin O (2017) Stereoscopic particle image velocimetry investigations of the mixed convection exchange flow through a horizontal vent. Exp Fluids 58(10):1-10. https://doi.org/10.1007/s00348-017-2434-7

24. Ward-Smith AJ (1980) Internal fluid flow the fluid dynamics of flow in pipes and ducts. Oxford University Press, Oxford

25. Wise NH, Hunt GR (2020) Buoyancy-driven unbalanced exchange flow through a horizontal opening. J Fluid Mech. https://doi.org/10.1017/jfm.2020.55

26. Wood IR (1968) Selective withdrawal from a stably stratified fluid. J Fluid Mech 32(2):209-223. https://doi.org/10.1017/S0022112068000686

Publisher's Note Springer Nature remains neutral with regard to jurisdictional claims in published maps and institutional affiliations. 